\title{
GC-MS analysis and in silico activity prediction of phytocompounds in the roots of Chrysopogon zizanioides (L.) Roberty
}

\author{
Shanti Vasudevan C N \& I'ma Neerakkal \\ Department of Botany, Sacred Heart College, Thevara 682 013, Kochi, Kerala, India \\ *Email: ima@shcollege.ac.in
}

\section{ARTICLE HISTORY}

Received: 25 October 2020

Accepted: 12 March 2021

Published: 24 March 2021

\section{KEYWORDS}

Chrysopogon zizanioides

Roots

Methanolic extract

GC-MS

Swiss ADME

\section{ABSTRACT}

Chrysopogon zizanioides (L.) Roberty (Poaceae) commonly known as Ramachamis an aromatic, vigorous growing perennial grass with medicinal properties. The plant is tolerant to extreme soil and climatic conditions and is known for its cooling properties. Roots of the plant are widely used as body scrubber and is suggested for skin diseases in Ayurveda. The present work aims to identify the components in the crude methanolic root extract of $C$. zizanioides using GC-MS and also to predict the pharmacokinetic behaviour of selected compounds in silico using Swiss ADME online server . 41 compounds were identified of which sesquiterpenes formed the major group. Sesquiterpene Vetivenic acid was the compound with a maximum peak area of $38.9 \%$. Components identified is reported to possess a range of biological activities like anti oxidant, antibacterial, anti cancer, anti inflammatory, anti ulcer, analgesic and insecticidal activities. Compounds with higher peak area like Vetivenic acid, beta vatirenene, beta.-Cedren-9-.alpha.-ol, D Viridiflorol, Gamma muurolene, (Z,E)-alpha-farnesene, Nootkatone, Aromadendrene oxide-(2), 7-Acetyl-2-hydroxy-2methyl-5isopropylbicyclo[4.3.0] nonane, Rosifoliol, 9,10-dehydro isolongifolene, Ylangenol, 4,7,10,13,16,19-Docosahexaenoic acid methyl ester, Carbonic acid, propargyl 2,2,2-tri chloroethyl ester, Oxacyclotetradeca-4,11-diyne, beta eudesmol and longifolene were evaluated in silico. All these compounds proved to obey Lipinski's rule-of-five and were water soluble. Vetivenic acid showed a good bioavailability score of $85 \%$ while the others showed $55 \%$. None of the compounds were substrates to P glycoprotein. The values predicted may be used for preliminary evaluation of pharmacological properties of $C$. zizanioides and also as monographs for the development of potential semisynthetic or synthetic drugs.

\section{Introduction}

Chrysopogon zizanioides (L.) Roberty is a medicinally useful plant known since ancient times. It is a perennial grass belonging to family Gramineae. Oil from the root of the plant have been used by the people for centuries. The plant is tolerant to extreme soil and climatic conditions and is known for its cooling properties (1). Roots of the plant are useful for hyperdipsia, burning sensation, skin diseases, nausea, vomiting, dyspepsia, flatulence, flatulence, bilious fever, gout, lumbago, sprains, halitosis, cephalalgia, amentia, amenorrhoea, helminthiasis and general debility (2).

Few Ayurvedic preparations from the roots of khus (C. zizanioides) are particularly used in relieving sense of heat and thus alleviating the symptoms of dermatoses (3). Volatile oils from different plant parts are known to improve flexibility of skin, have skin permeability, emollience, anti-inflammatory property and are effective against various skin ailments $(4,5)$.

GC-MS technique has been commonly used in $C$. zizanioides to identify the components in the volatile oil of roots. The present study attempt to use crude root extract obtained by maceration method for GCMS analysis. Maceration method is simple, cheap and less time consuming than the oil isolation methods.

Medicinal plants play a significant part in drug discovery, for the creation of novel bioactive compounds. The majority of the drugs endorsed for clinical trials are either natural Products or their analogs (6). The fact that increasing number of drugs don't reach the market because of their low

(C) Shanti V C N \& Neerakkal I (2021). This is an open-access article distributed under the terms of the Creative Commons Attribution License, which permits unrestricted use, distribution and reproduction in any medium, provided the original author and source are credited (https://creativecommons.org/licenses/by/4.0/).

To cite this article: Shanti V C N, Neerakkal I. GC-MS analysis and in silico activity prediction of phytocompounds in the roots of Chrysopogonzizanioides (L.) Roberty. Plant Science Today. 2021;8(1):218-224. https://doi.org/10.14719/pst.2021.8.1.1008 
pharmacokinetic profiles has necessitated the study of pharmacokinetic properties at early stages of drug development programmes (7).

An ideal drug needs to satisfy properties like easy oral absorption, easy transportation to the desired site of action, inability to form toxic products before reaching the targeted site of action and easy elimination from the body. All these properties are often referred to as ADME (absorption, distribution, metabolism and elimination) properties or ADMET (when toxicity assessment is included).

Use of computer-based strategies in the prediction of ADMET properties of drug leads at beginning stages of drug discovery are becoming increasingly popular being less expensive and less time consuming. Now a days various computational tools are widely used for predicting these pharmacokinetic properties. of the several computational tools available for the prediction of pharmacokinetic properties, Swiss ADME online tool provides free access to different predictive models for pharmacokinetics, physicochemical properties, medicinal chemistry friendliness and drug-likeness of compounds (8).

The present study was aimed to evaluate the volatile components in the crude methanolic root extract of $C$. zizanioides using GC-MS and also to predict the pharmacokinetic behaviour of selected compounds in silico using Swiss ADME online server.

\section{Materials and Methods}

\section{Plant collection and extraction}

The root sample of $C$. zizanioides were obtained from Aromatic and Medicinal Plant Research Station (AMPRS), Odakkali (Ernakulam district), Kerala, India. The sample was identified, authenticated and submitted (voucher specimen - Accession No: 16687 in the Herbarium of Kerala Forest Research Institute,
Thrissur, Kerala. The sample was washed thoroughly, dried under shade and ground to a fine powder in an electrical blender. The crude extract of $C$. zizanioides root was prepared using maceration technique by extracting $10 \mathrm{gm}$ of powdered sample in $50 \mathrm{ml}$ methanol in stoppered glass containers kept on a rotary shaker for $48 \mathrm{hrs}$ (9).

\section{GC-MS analysis}

The methanolic extract obtained from roots of $C$. zizanioides were analysed by GC-MS (Agilent, USA) using a High Performance-5MS 5\% Phenyl Methyl Silox capillary column (30m length x $250 \mu \mathrm{m}$ I.d. x $0.25 \mu \mathrm{m}$ film thickness). Electron impact system had an ionization energy of $70 \mathrm{eV}$ with a source temperature of $280{ }^{\circ} \mathrm{C}$. High purity helium gas was used at a constant flow rate of $1 \mathrm{ml} / \mathrm{min}$. The temperature of the oven was initially kept at $40{ }^{\circ} \mathrm{C}$ for $5 \mathrm{~min}$ then ramped at $5{ }^{\circ} \mathrm{C} / \mathrm{min}$ to $280{ }^{\circ} \mathrm{C}$ and finally increased to $325{ }^{\circ} \mathrm{C}$. Injection volume of $2 \mu \mathrm{l}$ was employed (split ratio 1:25). Injector temperature was maintained at $220^{\circ} \mathrm{C}$. The MS specifications were as follows: Ion source temperature: $230{ }^{\circ} \mathrm{C}$, interface temp: $300{ }^{\circ} \mathrm{C}$ scan range: $40-800 \mathrm{~m} / \mathrm{z}$, event time: $0.5 \mathrm{~s}$, solvent delay: 3 min. Positive electron impact ionization (EI) modes were used and data were collected using single ion monitoring (SIM). The mass spectra of the volatile components obtained through analysis were identified by comparing their mass spectra with the MS data library of National Institute of the Standards and Technology for The mass spectra of the volatile components obtained through analysis were identified by comparing their mass spectra with the MS data library of National Institute of Standards and Technology for identification of bioactive compounds (NIST 08.L) attached to the GCMS instrument and the results were obtained. Identified compounds were tabulated. Based on the reported biological activity of the phytocomponents analysed, compounds showing common pharmacological action were tabulated Fig 1.

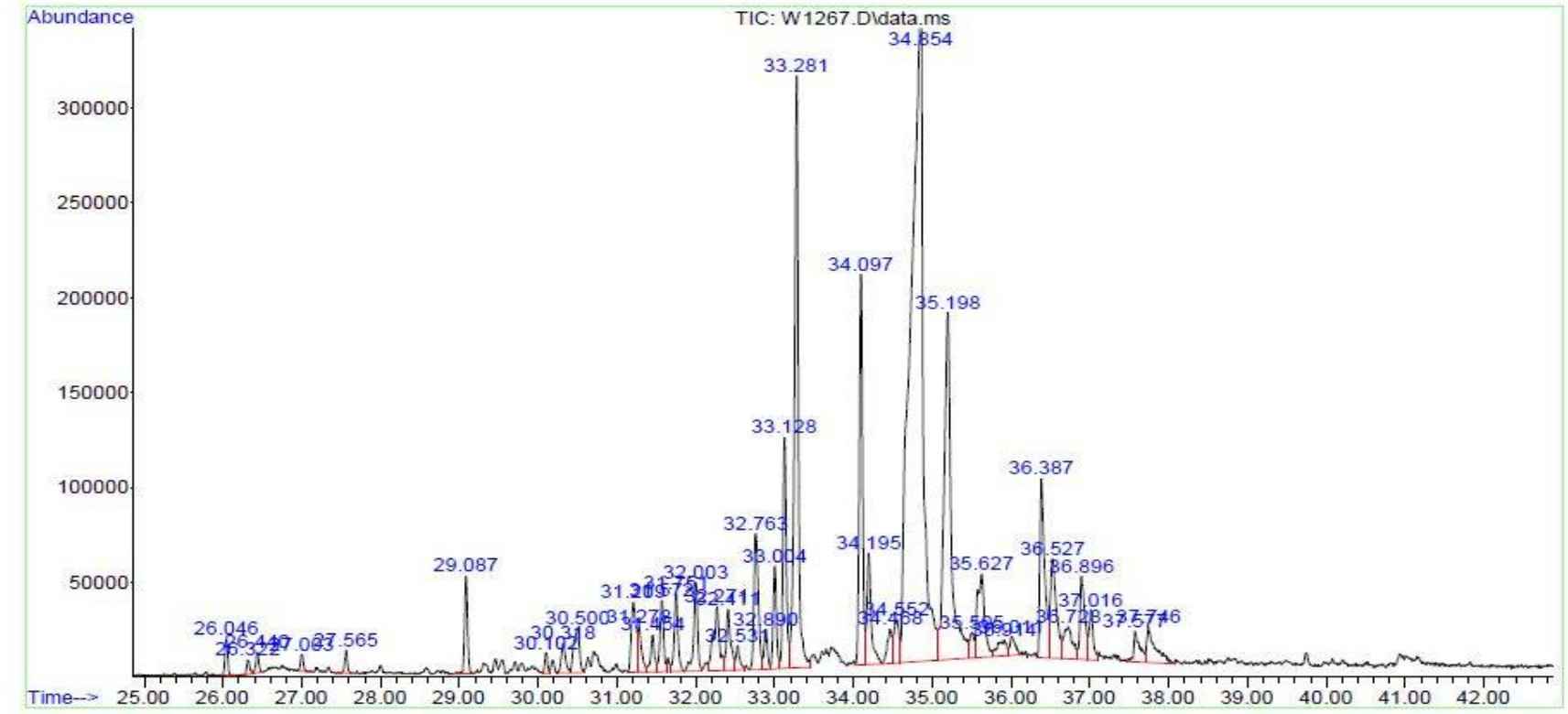

Fig. 1. GC-MS Chromatogram of $C$. zizanioides root methanolic extract. 


\section{ADME prediction}

Compounds with higher peak area were evaluated in silico. The molecular structure of the compounds were retrieved in ".sdf" format from PubChem and were used for ADME prediction using Swiss ADME online server (http://www.swissadme.ch) of Swiss institute of bioinformatics. Computational analyses were performed to predict the core pharmacokinetics parameters such as molecular weight, lipophilicity, water solubility, gastrointestinal absorption, Blood Brain Barrier permeability, P-glycoprotein substrate and skin permeability, drug likeliness and bioavailability score. After all analyses were completed, the graphical output was obtained in the form of "BOILED - Egg” model.

\section{Results}

\section{Chemical composition of extract by GC-MS analysis}

GC-MS chromatogram of the phytocomponents detected in the methanolic and root extract of $C$. zizanioides are shown in Table 1. The GC-MS analysis of methanolic root extract of $C$. zizanioides indicated the presence of forty-onecompounds. The active principles with their retention time, peak area (\%) and uses are presented in Table 2. Among the compounds identified, twenty-four compounds were sesquiterpenoids, two were monoterpenoids, two were esters, three were hydrocarbons and one was fatty acid. Cyclopentane acetaldehyde2-formyl-3methyl-.alpha.-methylene- (dolichodial) and ocimene were the monoterpenes identified. Sesquiterpene, 1H-3a, 6-Methanoazulene-3carboxylic acid, octahydro-7, 7-dimethyl-8-methylene-[3S-(3.alpha., 3a.alpha., 8a.alpha.)]-(Vetivenic acid / Khusenic acid) was the major compound identified in the extract with a peak area of $38.9 \%$ followed by beta.Ethylphenethyl alcohol (12.73\%), beta.-Vatirenene (7.24\%), beta.-Cedren-9-.alpha.-ol (4.45\%), $1 \mathrm{H}-$ Cycloprop[e] azulen-7-ol decahydro-1,1,7- trimethyl-4methylene [1ar-1a.alpha.,4a.alpha.,7.beta., 7b.alpha.)]- (Spthulenol / espatulenol) (3.31\%), alpha.-Farnesene (2.61 \%), 2(3H)-Naphthalenone, 4.4a,5,6,7,8-hexahydro-4,4a-dimethyl-6-(1-

methylethenyl)-,[4R-(4,alpha.,4a.alpha.,6.beta.)]-

(Nootkatone) (2.47\%) etc.

\section{In silico ADME evaluation}

Compounds with higher peak area like Vetivenic acid, beta vatirenene, beta.-Cedren-9-.alpha.-ol, D Viridiflorol, Gamma muurolene, (Z, E)-alphafarnesene, Nootkatone, Aromadendrene oxide-(2), 7Acetyl-2-hydroxy-2-methyl-5-isopropylbicyclo[4.3.0] nonane, Rosifoliol, 9,10-dehydro isolongifolene, Ylangenol, 4,7,10,13,16,19-Docosahexaenoic acid

Table 1. Phytocompounds detected in the methanolic root extract of $C$. zizanioides by GC-MS

\begin{tabular}{|c|c|c|c|}
\hline Sl. No. & Compound & RT & \\
\hline \multicolumn{4}{|c|}{ Monoterpene } \\
\hline 1 & Cyclopentane acetaldehyde2-formyl-3-methyl-.alpha.-methylene-[DOLICHODIAL] & 32.531 & 0.57 \\
\hline 2 & Ocimene & 32.89 & 0.74 \\
\hline \multicolumn{4}{|c|}{ Sesquiterpene } \\
\hline 3 & Gamma Himachalene & 26.046 & 0.57 \\
\hline 4 & $\begin{array}{l}\text { Naphthalene, 1,2,3,4,4a,8a-hexahydro-4,7-dimethyl1-(1-methylethyl)-naphthalene } \\
\text { [ALPHA AMORPHENE] }\end{array}$ & 26.439 & 0.46 \\
\hline 5 & alpha.-Cubebene & 27.004 & 0.54 \\
\hline 6 & 1,3,6,10-Dodecatetraene, 3,7,11-trimethyl-, (Z,E)- [(Z,E)-ALPHA-FARNESENE] & 27.340 & 0.19 \\
\hline 7 & cyclohexene 3-methyl-6-(1-methylethenyl)- (3r-trans)- [TRANS LIMONENE] & 27.565 & 0.42 \\
\hline 8 & $\begin{array}{l}\text { 1H-Cycloprop[e]azulene, decahydro 1,1,7-trimethyl-4-methylene-,[1aR } \\
\text { (1a.alpha.,4a.beta.,7.alpha.,7a.beta.,7b.alpha.)]- } \\
\text { [AROMADENDRENE] }\end{array}$ & 28.001 & 0.21 \\
\hline 9 & $\begin{array}{l}\text { 2-Naphthalenemethanol, 2,3,4,4a,5,6,7,8-octahydro.alpha.,.alpha.,4a,8-tetramethyl[2R- } \\
\text { (2.alpha.,4a.beta.,8.beta.)]- }\end{array}$ & 29.087 & 1.61 \\
\hline 10 & Caryophyllene oxide & 29.459 & 0.56 \\
\hline 11 & alpha.-Bisabolol & 29.792 & 0.24 \\
\hline 12 & $\begin{array}{l}\text { Naphthalene, 1,2,3,4,4a,5,6,8a-octahydro-4a,8-dimethyl-2-(1-methylethenyl[2R- } \\
\text { (2.alpha.,4a.alpha.,8a.beta.)]- [AROMADENDRENE] }\end{array}$ & 30.102 & 0.31 \\
\hline 13 & 2,6,10-Dodecatrien-1-ol, 3,7,11-trimethyl-, (Z,E)- [FARNESOL] & 30.318 & 0.71 \\
\hline 14 & $\begin{array}{l}\text { 1,4-Methanoazulene, decahydro-4,8,8-trimethyl-9-methylene-, [1S- } \\
\text { (1.alpha.,3a.beta.,4.alpha.,8a.beta.)]- [LONGIFOLENE] }\end{array}$ & 30.5 & 1.44 \\
\hline 15 & $\begin{array}{l}\text { 2-Naphthalenemethanol, decahydro alpha.,.,alpha.,4a-trimethyl-8-methylene-, [2R- } \\
\text { (2.alpha.,4a.alpha.,8a.beta.)]- [BETA EUDESMOL] }\end{array}$ & 30.714 & 1.05 \\
\hline 16 & $\begin{array}{l}\text { Naphthalene, 1,2,3,4,4a,5,6,8a-octahydro-7-methyl-4-methylene-1-(1-methylethyl)-, } \\
\text { ( 1.alpha.,4a.alpha., 8a.alpha.)- [GAMMA MUUROLENE] }\end{array}$ & 31.211 & 2.83 \\
\hline 17 & $\begin{array}{l}\text { 2(3H)-Naphthalenone, 4,4a,5,6,7,8- hexahydro-4,4a-dimethyl-6-(1-methylethenyl)-, [4R- } \\
\text { (4.alpha.,4a.alpha.,6.beta.)]- [NOOTKATONE] }\end{array}$ & 31.571 & 2.47 \\
\hline 18 & Isolongifolene 9,10-dehydro & 31.751 & 1.61 \\
\hline 19 & Aromadendrene oxide-(2) & 32.002 & 1.97 \\
\hline 20 & Tricyclo[4.4.0.02,7]dec-3-ene-3-methanol, 1-methyl-8-(1-methylethyl)-, [YLANGENOL] & 32.41 & 1.53 \\
\hline 21 & $\begin{array}{l}\text { 1H-Cycloprop[e]azulen-7-ol decahydro-1,1,7-trimethyl-4-methylene [1ar- } \\
\text { (1a.alpha.,4a.alpha.,7.beta., 7a.beta.,7b.alpha.)]- [SPTHULENOL / ESPATULENOL] }\end{array}$ & 32.763 & 3.31 \\
\hline 22 & .beta.-Cedren-9-.alpha.-ol & 33.128 & 4.45 \\
\hline 23 & $\begin{array}{l}\text { 1H-Cycloprop[e]azulen-4-oldecahydro-1,1,4,7-tetramethyl-, [1ar- } \\
\text { (1a.alpha.,4.beta.,4a.beta.,7.alpha., 7a.beta.,7b.alpha.)]- [D VIRIDIFLOROL] }\end{array}$ & 33.494 & 0.36 \\
\hline 24 & beta.-Vatirenene & 34.097 & 7.24 \\
\hline 25 & alpha.-Farnesene & 34.195 & 2.61 \\
\hline
\end{tabular}




\begin{tabular}{|c|c|c|c|}
\hline 26 & $\begin{array}{l}\text { 1H-3a,6-Methanoazulene-3-carboxylic acid, octahydro-7,7-dimethyl-8-methylene- [3S- } \\
\text { (3.alpha.,3a.alpha.,6.alpha.,8a.alpha.)]- [VETIVENIC ACID / KHUSENIC ACID ] }\end{array}$ & 34.854 & 38.9 \\
\hline \multicolumn{4}{|c|}{ Ester } \\
\hline 27 & 4,7,10,13,16,19-Docosahexaenoic acid, methyl ester(all-Z)- & 33.732 & 1.48 \\
\hline 28 & Carbonic acid, propargyl 2,2,2-tri chloroethyl ester & 33.732 & 1.48 \\
\hline \multicolumn{4}{|c|}{ Hydrocarbon } \\
\hline 29 & Toluene & 4.295 & 0.64 \\
\hline 30 & Hexane & 26.758 & 0.48 \\
\hline 31 & 3,4-Dimethoxytoluene & 29.323 & 0.36 \\
\hline \multicolumn{4}{|c|}{ Alcohol } \\
\hline 32 & beta.-Ethylphenethyl alcohol & 33.281 & 12.73 \\
\hline \multicolumn{4}{|c|}{ Fatty acid } \\
\hline 33 & 2-Nonynoic acid & 30.99 & 0.23 \\
\hline \multicolumn{4}{|c|}{ Others } \\
\hline 34 & 3-Aminopyrrolidine & 26.758 & 0.48 \\
\hline 35 & 1-Methoxy-1,4-cyclohexadiene & 29.928 & 0.28 \\
\hline 36 & Cyclopentane-3'-spirotricyclo[3.1.0.0(2,4)]hexane-6'-spirocyclopentane & 30.186 & 0.22 \\
\hline 37 & 1,4-Methanoazulenedecahydro-4,8,8-trimethyl-9-methylen & 30.496 & 1.44 \\
\hline 39 & Cyclopropane1,1-dichloro-2,2,3,3 -tetramethyl- & 32.27 & 2.27 \\
\hline 40 & 7-Acetyl-2-hydroxy-2-methyl-5-isopropylbicyclo[4.3.0]nonane & 33.004 & 1.82 \\
\hline 41 & Oxacyclotetradeca-4,11-diyne & 34.549 & 1.35 \\
\hline
\end{tabular}

Table 2. Reported pharmacological activities of compounds identified in C. zizanioides roots

\begin{tabular}{|c|c|c|}
\hline Sl. No. & Pharmacological Activities & C. zizanioides \\
\hline 1 & Anicancer, AntianoxicAnti ulcer, Hepatoprotective, Pesticide & Beta eudesmol (18) \\
\hline 2 & Antiinflammatory & $\begin{array}{l}\text { alpha.-Cubebene (19) } \\
\text { alpha.-Bisabolol (20) } \\
\text { Spathulenol (21) } \\
\text { D Viridiflorol (22) } \\
\text { Caryophyllene oxide (23) }\end{array}$ \\
\hline 3 & Antimicrobial & $\begin{array}{l}\text { beta.-Vatirenene (24) } \\
\text { Ocimene (25) } \\
\text { gamma himachelene (26) } \\
\text { alpha.-Cubebene (19) } \\
\text { alpha.-Bisabolol (27) } \\
\text { Spathulenol (21) } \\
\text { Vetivenic acid (28) } \\
\text { 2-Nonynoic acid (29) } \\
\text { D Viridiflorol (22) } \\
\end{array}$ \\
\hline 4 & Antioxidant & $\begin{array}{l}\text { D Viridiflorol (22) } \\
\text { Isolongifolene 9,10-dehydro (30) } \\
\text { Spathulenol (21) }\end{array}$ \\
\hline 5 & Skin problems & $\begin{array}{l}\text { alpha.-Bisabolol (27) } \\
\text { aromadendrene (31) }\end{array}$ \\
\hline 6 & Anticancer & $\begin{array}{l}\text { Caryophyllene oxide (18) } \\
\text { Aromadendrene (18) }\end{array}$ \\
\hline 7 & Antiulcer & $\begin{array}{l}\text { Beta eudesmol (18) } \\
\text { alpha.-Bisabolol (27) } \\
\text { Nootkatone (18) }\end{array}$ \\
\hline 8 & Perfumes & $\begin{array}{l}\text { Ylangenol (32) } \\
\text { alpha.-Farnesene (33) } \\
\text { Ocimene (18) } \\
\text { alpha.-Bisabolol (27) }\end{array}$ \\
\hline 9 & Flavour & Farnesol (18) \\
\hline 10 & Insecticide & $\begin{array}{l}\text { Dolichodial (18) } \\
\text { Ocimene (25) } \\
\text { Nootkatone (34) } \\
\text { alpha.-Farnesene (33) }\end{array}$ \\
\hline
\end{tabular}

methyl ester, Carbonic acid, propargyl 2,2,2-tri chloroethyl ester, Oxacyclotetradeca-4,11-diyne, beta eudesmol and longifolene were evaluated in silico. The observed values are tabulated (Table 3 ) and the graphical output in the form of "BOILED-egg" model is represented (Fig. 2). All the studied compounds proved to obey Lipinski's rule-of-five and were water soluble. Vetivenic acid showed a good bioavailability score of $85 \%$ while the others showed $55 \%$. None of the compounds were substrates to $\mathrm{P}$ glycoprotein.

\section{Discussion}

C. zizanioides root are a rich source of bioactive compounds. GC-MS analysis of the crude root extract of $C$. zizanioides in methanol, indicated the presence of sesquiterpenes, monoterpenes, esters, alcohols and fatty acids. Of the 41 compounds identified, sesquiterpenes were the major group. Vetivenic acid was the compound identified with major peak area. The presence of gamma himachelene, nootkatone, aromadendrene oxide, vetivenic acid, betavetirene, alpha farnesene, alpha bisabolol, gamma murolene, 
Table 3. Result of ADME prediction of phytoconstituents identified in GC-MC of methanolic root extract of $C$. zizanioides

\begin{tabular}{|c|c|c|c|c|c|c|c|c|c|c|}
\hline $\begin{array}{l}\text { Sl. } \\
\text { No. }\end{array}$ & Compound & $\begin{array}{l}\text { Molecula } \\
\text { r mass }\end{array}$ & $\begin{array}{l}\text { Lipophili } \\
\text { city }\end{array}$ & $\begin{array}{l}\text { Water } \\
\text { Solubil } \\
\text { ity }\end{array}$ & $\begin{array}{l}\text { GI } \\
\text { absorp } \\
\text { tion }\end{array}$ & $\begin{array}{c}\text { BBB } \\
\text { permeab } \\
\text { ility }\end{array}$ & $\begin{array}{l}\text { P-gp } \\
\text { substr } \\
\text { ate }\end{array}$ & $\begin{array}{l}\text { Skin } \\
\text { permeatio } \\
\text { n } \\
\text { cm/s }\end{array}$ & $\begin{array}{l}\text { Drug } \\
\text { likeline } \\
\text { ss }\end{array}$ & $\begin{array}{l}\text { Bio } \\
\text { availabili } \\
\text { ty score }\end{array}$ \\
\hline 1 & $\begin{array}{l}\text { 1H-3a,6-Methanoazulene-3-carboxylic acid, } \\
\text { octahydro-7,7-dimethyl-8-methylene- [3S- } \\
\text { (3.alpha.,3a.alpha.,6.alpha.,8a.alpha.)]- } \\
\text { [VETIVENIC ACID / KHUSENIC ACID ] }\end{array}$ & 234.33 & 3.21 & Soluble & High & Yes & No & -5.15 & Yes & 0.85 \\
\hline 2 & Beta Vatirenene & 202.34 & 4.13 & Soluble & Low & No & No & -4.38 & Yes & 0.55 \\
\hline 3 & .beta.-Cedren-9-.alpha.-ol & 220.35 & 3.35 & Soluble & High & Yes & No & -5.12 & Yes & 0.55 \\
\hline 4 & $\begin{array}{l}\text { 1H-Cycloprop[e]azulen-7-ol decahydro-1,1,7- } \\
\text { trimethyl-4-methylene [1ar- } \\
\text { (1a.alpha.,4a.alpha.,7.beta., } \\
\text { 7a.beta.,7b.alpha.)]- [SPTHULENOL / } \\
\text { ESPATULENOL] }\end{array}$ & 222.37 & 3.43 & Soluble & High & Yes & No & -5.00 & Yes & 0.55 \\
\hline 5 & $\begin{array}{l}\text { Naphthalene, 1,2,3,4,4a,5,6,8a-octahydro-7- } \\
\text { methyl-4-methylene-1-(1-methylethyl)-, } \\
\text { ( 1.alpha.,4a.alpha., 8a.alpha.)- [GAMMA } \\
\text { MUUROLENE] }\end{array}$ & 204.35 & 4.17 & Soluble & Low & No & No & -4.49 & Yes & 0.55 \\
\hline 6 & alpha.-Farnesene & 204.35 & 4.96 & Soluble & Low & No & No & -3.20 & Yes & 0.55 \\
\hline 7 & $\begin{array}{l}\text { 2(3H)-Naphthalenone, 4,4a,5,6,7,8- } \\
\text { hexahydro-4,4a-dimethyl-6-(1- } \\
\text { methylethenyl)-, [4R- } \\
\text { (4.alpha.,4a.alpha.,6.beta.)]- [NOOTKATONE] }\end{array}$ & 218.33 & 3.57 & Soluble & High & Yes & No & -4.89 & Yes & 0.55 \\
\hline 8 & Aromadendrene oxide-(2) & 220.35 & 3.54 & Soluble & High & Yes & No & -5.03 & Yes & 0.55 \\
\hline 9 & $\begin{array}{l}\text { 7-Acetyl-2-hydroxy-2-methyl-5- } \\
\text { isopropylbicyclo[4.3.0]nonane }\end{array}$ & 238.37 & 2.8 & Soluble & High & Yes & No & -5.94 & Yes & 0.55 \\
\hline 10 & $\begin{array}{l}\text { 2-Naphthalenemethanol, 2,3,4,4a,5,6,7,8- } \\
\text { octahydro.alpha.,.alpha.,4a,8- } \\
\text { tetramethyl[2R-(2.alpha.,4a.beta.,8.beta.)]- } \\
\text { (Rosifoliol) }\end{array}$ & 222.37 & 3.58 & Soluble & High & Yes & No & -4.90 & Yes & 0.55 \\
\hline$\overline{11}$ & Isolongifolene 9,10-dehydro & 202.34 & 4.18 & Soluble & Low & No & No & -4.01 & Yes & 0.55 \\
\hline 12 & $\begin{array}{l}\text { Tricyclo[4.4.0.02,7]dec-3-ene-3-methanol, 1- } \\
\text { methyl-8-(1-methylethyl)-,[YLANGENOL] }\end{array}$ & 220.35 & 3.26 & Soluble & High & Yes & No & -5.36 & Yes & 0.55 \\
\hline 13 & $\begin{array}{l}\text { 4,7,10,13,16,19-Docosahexaenoic acid, } \\
\text { methyl ester(all-Z)- }\end{array}$ & 324.51 & 6.32 & $\begin{array}{l}\text { Moder } \\
\text { ately } \\
\text { soluble }\end{array}$ & Low & No & No & -3.77 & Yes & 0.55 \\
\hline 14 & $\begin{array}{l}\text { Carbonic acid, propargyl 2,2,2-tri } \\
\text { chloroethyl ester }\end{array}$ & 231.46 & 2.21 & Soluble & High & Yes & No & -5.94 & Yes & 0.55 \\
\hline 15 & Oxacyclotetradeca-4,11-diyne & 190.28 & 3.21 & Soluble & High & Yes & No & -5.01 & Yes & 0.55 \\
\hline 16 & $\begin{array}{l}\text { 2-Naphthalenemethanol, decahydro } \\
\text { alpha.,.alpha.,4a-trimethyl-8-methylene-, } \\
\text { [2R-(2.alpha.,4a.alpha.,8a.beta.)]- [BETA } \\
\text { EUDESMOL] }\end{array}$ & 222.37 & 3.61 & Soluble & High & Yes & No & -5.00 & Yes & 0.55 \\
\hline 17 & $\begin{array}{l}\text { 1,4-Methanoazulene, decahydro-4,8,8- } \\
\text { trimethyl-9-methylene-, [1S- } \\
\text { (1.alpha.,3a.beta.,4.alpha.,8a.beta.)]- } \\
\text { [LONGIFOLENE] }\end{array}$ & 204.35 & 4.50 & Soluble & Low & No & No & -3.94 & Yes & 0.55 \\
\hline
\end{tabular}

beta eudesmol were identified. Similar findings were reported earlier in the essential oil of $C$. zizanioides $(10-12)$. But the methodology followed in this study is simpler and cheaper than other distillation methods followed (13).

Reported pharmacological action of the compounds identified (Table 2) supports the suggested medicinal use of root of $C$. zizanioides in Ayurveda (2). The findings support the traditional use of roots of $C$. zizanioides (well known as Ramacham) as body scrubber.

Of the 41 compounds identified, compounds with ADME information and peak area above 1\% were tabulated.17 compounds thus tabulated showed molecular weight less than 500 Daltons which indicated ability of trans cutaneous permeation for these compounds. Topical dermatological therapy focusses on development of innovative compounds under 500 daltons (14). All the 17 compounds selected satisfied the Lipinski rule of five indicating drug likeliness. All the studied compounds in $C$. zizanioides showed water solubility thus favouring more bioavailability. This finding is an added advantage as more than $40 \%$ new chemical entities developed in pharmaceutical industry are known to be practically insoluble in water. Solubility has become major challenge for formulation scientist. Any drug to be absorbed must be present in the form of solution at the site of absorption (15). Carbonic acid, propargyl 2,2,2-tri chloroethyl ester and 7-Acetyl-2-hydroxy-2methyl-5-isopropylbicyclo [4.3.0] nonane showed log P (lipophilicity) less than 2 indicating lower toxicity (16). None of the compounds were substrates to P- 


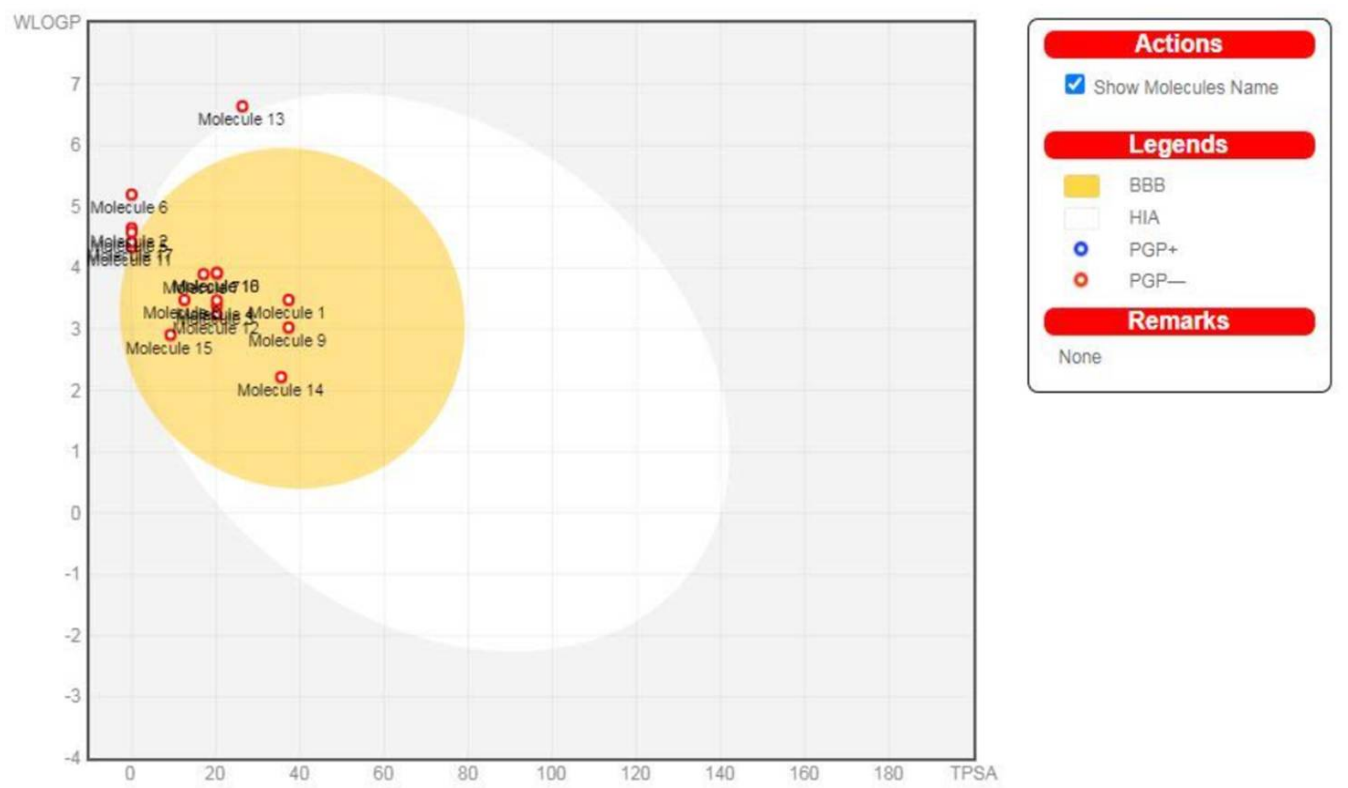

Fig. 2. Boiled Egg Model of the Phytoconstituents of $C$. zizanioides roots.

glycoprotein and thus indicating a better bio availability as reported previously (17). The graphical output of the analysed parameters in the form of "BOILED-Egg” gives a global evaluation about passive absorption (inside/outside the white), passive brain access (inside/outside the yolk) and active efflux from the CNS or to the gastrointestinal lumen by colourcoding: blue dots for P-gp substrates (PGP+) and red dots for P-gp non-substrate (PGP-) (Fig. 2) (8).

Thus, the study shows that many of the volatile components present in the oil of $C$. zizanioides can be extracted by simple maceration method of extraction using methanol. Among the identified compounds, compounds with ADME information indicated transcutaneous permeation, proved to obey Lipinski's rule-of-five and were water soluble. Vetivenic acid showed a good bioavailability score of $85 \%$ while the others showed $55 \%$. None of the compounds were substrates to $\mathrm{P}$ glycoprotein. The pharmacokinetic parameters studied indicate drug likeliness for the studied compounds but require further in vitro and in vivo studies for validation. The values predicted may be used for preliminary evaluation of pharmacological properties of $C$. zizanioides and also as monographs for the development of potential semisynthetic or synthetic drugs.

\section{Acknowledgements}

The corresponding author thank UGC for granting Teacher Fellowship under Faculty Development Programme.

\section{Authors' contributions}

SVCN carried out the experiments and wrote the entire manuscript. IN gave overall direction and helped in interpreting the results.

\section{Conflict of interests}

The authors declare no conflict of interest.

\section{References}

1. Lim TK. Chrysopogon zizanioides. In: Edible Medicinal and Non-Medicinal Plants. Springer, Cham. 2016;197-227. https://doi.org/10.1007/978-3-319-26062-4_16

2. Warrier PK, Nambiar VPK. Indian Medicinal Plants: A Compendium of 500 Species. $5^{\text {th }}$ vol. Chennai: Orient Longman Private Limited;2005;

3. Sehgal VN, Srivastava G. Traditional/ayurvedic pharmacotherapy of skin diseases. Skinmed. 2010;8(5):282-84.

4. Ali B, Al-Wabel NA, Shams S, Ahamad A, Khan SA, Anwar F. Essential oils used in aromatherapy: A systemic review. Asian Pacific Journal of Tropical Biomedicine. 2015;5(8):601-11. https://doi.org/10.1016/j.apjtb.2015.05.007

5. Aburjai T, Natsheh FM. Plants used in cosmetics. Phytotherapy Research: An International Journal Devoted to Pharmacological and Toxicological Evaluation of Natural Product $\quad$ Derivatives. 2003;17(9):987-1000. https://doi.org/10.1002/ptr.1363

6. Aswad M, Rayan M, Abu-Lafi S, Falah M, Raiyn J, Abdallah Z, Rayan A, et al. Nature is the best source of anti-inflammatory drugs: Indexing natural products for their anti-inflammatory bioactivity. Inflammation Research. 2018;67(1):67-75 https://doi.org/10.1007/s00011-017-1096-5

7. Lucas AJ, Sproston JL, Barton P, Riley RJ. Estimating human ADME properties, pharmacokinetic parameters and likely clinical dose in drug discovery. Expert opinion on drug discovery. https://doi.org/10.1080/17460441.2019.1660642

8. Daina A, Michielin O, Zoete V. SwissADME: a free web tool to evaluate pharmacokinetics, drug-likeness and medicinal chemistry friendliness of small molecules. Scientific Reports. 2017;7:42717. https://doi.org/10.1038/srep42717

9. Gomathi D, Kalaiselvi M, Ravikumar G, Devaki K, Uma C. GC MS analysis of bioactive compounds from the whole plant ethanolic extract of Evolvulusalsinoides (L.) L. Journal of Food Science and Technology. 2015;52(2):1212-27. https://doi.org/10.1007/s13197-013-1105-9

10. Kannappan A, Gowrishankar S, Srinivasan R, Pandian SK, Ravi AV. Antibiofilm activity of Vetiveria zizanioides root extract against methicillin-resistant Staphylococcus aureus. Microbial Pathogenesis. https://doi.org/10.1016/j.micpath.2017.07.016

2017;110:313-24.

11. Chahal KK, Kaushal S, Sandhu AK. Chemical composition and biological properties of Chrysopogon zizanioides (L.) Roberty syn. Vetiveria zizanioides (L.) Nash-A Review. Indian Journal of Natural Products and Resources (IJNPR)[Formerly Natural Product Radiance (NPR)]. 2015;6(4):251-60. 
12. Lima GM, Quintans-Júnior LJ, Thomazzi SM, Almeida EM, Melo MS, Serafini MR, Cavalcanti SC, Gelain DP, Santos JP, Blank AF, Alves PB, et al. Phytochemical screening, antinociceptive and anti-inflammatory activities of Chrysopogon zizanioides essential oil. Revistabrasileira de Farmacognosia. 2012;22(2):443-50. https://doi.org/10.1590/S0102$695 X 2012005000002$

13. David A, Wang F, Sun X, Li H, Lin J, Li P, Deng G. Chemical composition, antioxidant and antimicrobial activities of Vetiveria zizanioides (L.) Nash Essential Oil Extracted by Carbon Dioxide Expanded Ethanol. Molecules. 2019;24(10):1897. https://doi.org/10.3390/molecules24101897

14. Bos JD, Meinardi MM. The 500 Dalton rule for the skin penetration of chemical compounds and drugs. Experimental Dermatology: Viewpoint. 2000;9(3):165-69. https://doi.org/10.1034/j.1600-0625.2000.009003165.x

15. Savjani KT, Gajjar AK, Savjani JK. Drug solubility: importance and enhancement techniques. ISRN pharmaceutics; 2012. https://doi.org/10.5402/2012/195727

16. Arnott JA, Planey SL. The influence of lipophilicity in drug discovery and design. Expert opinion on drug discovery. 2012;7(10):863-75.https://doi.org/10.1517/17460441.2012.714363

17. Leopoldo M, Nardulli P, Contino M, Leonetti F, Luurtsema G, Colabufo NA. An updated patent review on P-glycoprotein inhibitors (2011-2018). Expert Opinion on Therapeutic Patents. 2019;29(6):455-61.

https://doi.org/10.1080/13543776.2019.1618273

18. Duke J, Bogenschutz MJ. Dr. Duke's phytochemical and ethnobotanical databases. USDA, Agricultural Research Service; 1994

19. Lee SK, Kim SD, Lee HY, Baek SH, Ko MJ, Son BG, Park S, Choi YW, Bae YS, et al. a-Iso-cubebene, a natural compound isolated from Schisandra chinensis fruit, has therapeutic benefit against polymicrobial sepsis. Biochemical and Biophysical Research Communications. $2012 \quad$ Sep 21;426(2):226-31. https://doi.org/10.1016/j.bbrc.2012.08.070.

20. Rocha NF, Rios ER, Carvalho AM, Cerqueira GS, de Araújo Lopes A, Leal LK, Dias ML, de Sousa DP, de Sousa FC, et al. Anti-nociceptive and anti-inflammatory activities of (-)-abisabolol in rodents. Naunyn-Schmiedeberg's Archives of Pharmacology. https://doi.org/10.1007/s00210-011-0679-X 2011:384(6):525-33

21. do Nascimento KF, Moreira FM, Santos JA, Kassuya CA, Croda JH, Cardoso CA, do Carmo Vieira M, Ruiz AL, Foglio MA, de Carvalho JE, Formagio AS, et al. Antioxidant, antiinflammatory, antiproliferative and antimycobacterial activities of the essential oil of Psidium guineense Sw. and spathulenol. Journal of Ethnopharmacology. 2018 Jan 10;210:351-58. https://doi.org/10.1016/j.jep.2017.08.030.

22. Trevizan LN, do Nascimento KF, Santos JA, Kassuya CA, Cardoso CA, do Carmo Vieira M, Moreira FM, Croda J, Formagio AS, et al., Anti-inflammatory, antioxidant and antiMycobacterium tuberculosis activity of viridiflorol: The major constituent of Allophylus edulis (A. St.-Hil., A. Juss. \& Cambess.) Radlk. Journal of Ethnopharmacology. 2016;192:510-5. https://doi.org/10.1016/j.jep.2016.08.053
23. Chavan MJ, Wakte PS, Shinde DB. Analgesic and antiinflammatory activity of Caryophyllene oxide from Annona squamosa L. bark. Phytomedicine. 2010;17(2):149-51. https://doi.org/10.1016/j.phymed.2009.05.016

24. Koo S, Thomas HR, Comolli JC, Rearden P, Baden LR, Marty FM, Inventors; Charles Stark Draper Laboratory Inc, Brigham, Women's Hospital, Assignee. Diagnosis and treatment of invasive aspergillosis. United States patent US 10,031,125. 2018 Jul 24

25. Kishimoto K, Matsui K, Ozawa R, Takabayashi J. Volatile C6aldehydes and allo-ocimene activate defense genes and induce resistance against Botrytis cinerea in Arabidopsis thaliana. Plant and Cell Physiology. 2005;46(7):1093-102. https://doi.org/10.1093/pcp/pci122

26. Tarek N, Hassan HM, AbdelGhani SM, Radwan IA, Hammouda O, El-Gendy AO. Comparative chemical and antimicrobial study of nine essential oils obtained from medicinal plants growing in Egypt. Beni-Suef University Journal of Basic and Applied Sciences. 2014;3(2):149-56. https://doi.org/10.1016/j.bjbas.2014.05.009

27. Forrer M, Kulik EM, Filippi A, Waltimo T. The antimicrobial activity of alpha-bisabolol and tea tree oil against Solobacterium moorei, a Gram-positive bacterium associated with halitosis. Archives of Oral Biology. 2013;58(1):10-16 https://doi.org/10.1016/j.archoralbio.2012.08.001

28. Dwivedi GR, Gupta S, Roy S, Kalani K, Pal A, Thakur JP, Saikia D, Sharma A, Darmwal NS, Darokar MP, Srivastava SK, et al. Tricyclic sesquiterpenes from Vetiveria zizanoides (L.) Nash as antimycobacterial agents. Chemical Biology \& Drug Design. 2013 Nov;82(5):587-94. https://doi.org/10.1111/cbdd.12188

29. Hudak ES, Mercer BD, Wotiz JH. Fungistatic, bacteriostatic, and amebicidal studies on the isomeric normal nonynoic acids. Journal of the American Pharmaceutical Association. 1956;45(5):327-30. https://doi.org/10.1002/jps.3030450516

30. Rangasamy K, Namasivayam E. In vitro antioxidant and free radical scavenging activity of isolongifolene. Asian J Biol Sci. 2014;7(1):13-23.

31. Pavithra PS, Mehta A, Verma, R. S. Aromadendrene oxide 2, induces apoptosis in skin epidermoid cancer cells through ROS mediated mitochondrial pathway. Life Sciences. 2018;197:1929. https://doi.org/10.1016/j.lfs.2018.01.029

32. Gu JL, Li ZJ, Zhang HX, Du ZZ. Fragrant volatile sesquiterpenoids isolated from the essential oil of Laggeraptera odonta by using olfactory-guided fractionation. Chemistry \& Biodiversity. 2014;11(9):1398-405. https://doi.org/10.1002/cbdv.201400051

33. Rowan DD, Allen JM, Fielder S, Spicer JA, Brimble MA Identification of conjugated triene oxidation products of alphafarnesene in apple skin. Journal of Agricultural and Food Chemistry. https://doi.org/10.1021/jf00056a016

1995;43(8):2040-45

34. Leonhardt RH, Berger RG. Nootkatone. In: Biotechnology of Isoprenoids Springer, Cham; 2014.391-404. 